\title{
Electrochemically Induced pH Change: Time-Resolved Confocal Fluorescence Microscopy Measurements and Comparison with Numerical Model
}

\author{
Nakul Pande,* Shri K. Chandrasekar, Detlef Lohse, Guido Mul, Jeffery A. Wood, Bastian T. Mei,* \\ and Dominik Krug*
}

Cite This: J. Phys. Chem. Lett. 2020, 11, 7042-7048

Read Online

ACCESS | Lلll Metrics \& More | 回 Article Recommendations | st Supporting Information

ABSTRACT: Confocal fluorescence microscopy is a proven technique, which can image near-electrode $\mathrm{pH}$ changes. For a complete understanding of electrode processes, timeresolved measurements are required, which have not been achieved previously. Here we present the first measurements of time-resolved $\mathrm{pH}$ profiles with confocal fluorescence microscopy. The experimental results compare favorably with a one-dimensional reactiondiffusion model; this holds up to the point where the measurements reveal threedimensionality in the $\mathrm{pH}$ distribution. Specific factors affecting the $\mathrm{pH}$ measurement such as attenuation of light and the role of dye migration are also discussed in detail. The method is further applied to reveal the buffer effects observed in sulfate-containing electrolytes. The work presented here is paving the way toward the use of confocal fluorescence microscopy in the measurement of 3D time-resolved $\mathrm{pH}$ changes in numerous electrochemical settings, for example, in the vicinity of bubbles.

$\mathrm{E}$ lectrochemical reactions in aqueous solutions are strongly affected by the $\mathrm{pH}$ near the electrode. In corrosion science, potential $-\mathrm{pH}$ phase diagrams ${ }^{1}$ (Pourbaix diagrams) best summarize this relationship. Moreover, in applications of energy storage and material conversion (e.g., $\mathrm{CO}_{2}$ and $\mathrm{N}_{2}$ reduction to useful products), where protons in solution are consumed, there is a direct link between the $\mathrm{pH}$ and the efficiency of the electrochemical cell. Measuring and understanding $\mathrm{pH}$ profiles near electrodes is therefore essential and can provide insight into the local surface chemistry and help design efficient electrochemical systems. This is particularly relevant in the reduction of $\mathrm{CO}_{2}$, where sensitivity to the near electrode $\mathrm{pH}$ may limit the desired product formation. ${ }^{2-4}$

An effective technique to detect $\mathrm{pH}$ changes is the use of indicator molecules, such as fluorescein, whose fluorescence changes with $\mathrm{pH}$. Unlike point measurements, e.g., via scanning electrochemical microscopy, ${ }^{5}$ imaging fluorescence fields allows for spatially resolved $\mathrm{pH}$ measurement. When coupled with confocal microscopy, this approach offers an even higher spatial resolution and has already demonstrated its potential in electrochemical applications. ${ }^{6}$ For example, Unwin et al. $^{7,8}$ measured three-dimensional steady-state $\mathrm{pH}$ profiles on microelectrodes. Cannan et al. ${ }^{7}$ determined the $\mathrm{pH}$ change accompanied by the reduction of benzoquinone to hydroquinone. Similarly, Rudd et al. ${ }^{8}$ measured the $\mathrm{pH}$ profiles induced by the reduction of water and oxygen on gold electrodes. They considered different electrode shapes and compared their results with a steady-state reaction-diffusion model. Leenheer and Atwater ${ }^{9}$ applied the fluorescence method in a flow cell to compare the steady-state $\mathrm{pH}$ profiles formed (for hydrogen evolution) on patterned $\mathrm{Au}$ electrode surfaces. Furthermore, they measured $\mathrm{pH}$ profiles on various electrode materials, thereby suggesting this technique as a screening tool for identifying electrocatalysts. Leenheer and Atwater ${ }^{9}$ also they compared their measurements to a steadystate model, one including laminar flow.

Although fluorescent measurements of spatiotemporal $\mathrm{pH}$ profiles near ion-selective membranes have been recently undertaken ${ }^{10}$ with related electrokinetic modeling by Andersen et al., ${ }^{11}$ such measurements are lacking for electrolytic systems and near the electrodes. Here, besides electric field effects, large gradients in $\mathrm{pH}$ are created because of chemical reactions at the electrode surface. Time resolution is then essential to capture the dynamics at the electrode-electrolyte interface. One such application would be the measurement of $\mathrm{pH}$ profiles around growing hydrogen bubbles in solution, which may reveal transient reaction hot-spots. ${ }^{12,13}$ Similarly, other situations involving phase change, simultaneous electrode reactions, or bulk buffer reactions during electrolysis require time-resolved measurements for their accurate character-

Received: May 21, 2020

Accepted: July 28, 2020

Published: July 28, 2020 
ization. Certainly, a further development of time-resolved measurements techniques is urgently needed to understand dynamic processes occurring at electrode/electrolyte interfaces in electrochemical processes. In spite of the need, to the best of our knowledge, a quantitative comparison of time-resolved $\mathrm{pH}$ measurements and modeling using fluorescent dyes is not yet available in the literature. In this contribution we demonstrate the feasibility of using fluorescent dyes to measure spatiotemporally varying $\mathrm{pH}$ profiles in solution by comparing the $\mathrm{pH}$ changes arising from electrochemical oxygen reduction with a time-dependent reaction-diffusion model. We further apply this technique to highlight buffer effects in sulfatecontaining electrolytes.

Confocal Fluorescence $(p H)$ Microscopy. To carry out the measurements, an electrochemical cell assembly was mounted on top of an inverted confocal microscope. A schematic of the setup which also contains the relevant dimensions is shown in Figure 1a. The electrochemical housing was made of Teflon. In all measurements, a platinized titanium mesh was rolled up and placed as a ring at about $4 \mathrm{~cm}$ from the working electrode. This assembly successfully prevented any interference of the counter electrode reaction with the $\mathrm{pH}$ measurement. A $10 \mathrm{~nm}$ thick platinum film evaporated on a circular glass slide (thickness $170 \mu \mathrm{m}$, diameter $50 \mathrm{~mm}$ ) formed the working electrode. A $\mathrm{BASi} \mathrm{Ag} / \mathrm{AgCl}$ (in $3 \mathrm{M} \mathrm{NaCl}$ ) was used as a reference electrode. Unless otherwise stated, $0.5 \mathrm{M} \mathrm{NaClO}_{4}$ with $8 \mu \mathrm{M}$ sodium fluorescein was used as electrolyte. The measurements involved taking fluorescence images along the scanned direction $z$ (in a serial fashion as shown in Figure 1a). The mean of each image was then taken as the measured intensity at the corresponding $z$-position. The fluorescence signal was found to be compromised up to a distance $z \approx 100 \mu \mathrm{m}$ above the surface (see Experimental Section and the Supporting Information for further details). Therefore, any fluorescence intensity (and resulting $\mathrm{pH}$ ) information was obtained only above this threshold.

Fluorescein $(\mathrm{Fl})$ is a popular choice to probe $\mathrm{pH}$ changes in electrochemical cells. $^{7-9,14,15}$ The $\mathrm{pH}$ sensitivity of $\mathrm{Fl}$ is well documented $^{16-18}$ and arises from the existence of different protonated forms of the molecule in solution. The fluorescence emission of constant $\mathrm{pH}$ solutions was measured for the intensity-to-pH calibration (as shown in Figure $1 \mathrm{~b}$ ). These results were fitted with an analytical function to allow for a conversion from $\mathrm{Fl}$ intensities obtained in the experiments to $\mathrm{pH}$. The laser and confocal settings were kept constant throughout the study such that the curve in Figure $1 \mathrm{~b}$ applies to all experiments. The dye is found to be particularly $\mathrm{pH}$ sensitive in the range $5 \lesssim \mathrm{pH} \lesssim 10$ (indicated by the shading in Figure 1b), as evidenced by the pronounced increase of fluorescence emission intensity measured with increasing $\mathrm{pH}$ within this interval. However, the highly nonlinear intensity$\mathrm{pH}$ relationship suggests that measurement results beyond $\mathrm{pH} \gtrsim 8.5$ should be taken with caution. Details on the fit and the repeatability are provided in the Supporting Information. The effect of dye migration induced by the electric field has also been addressed therein. Migration is detrimental to the present technique, which assumes a homogeneous dye distribution. It was found that a certain minimum supporting electrolyte concentration is necessary to keep migration effects at bay.

Last, to compare with experimental results, we also simulated the $\mathrm{pH}$ profiles during reaction. Because the supporting electrolyte concentration is high, we adopted a

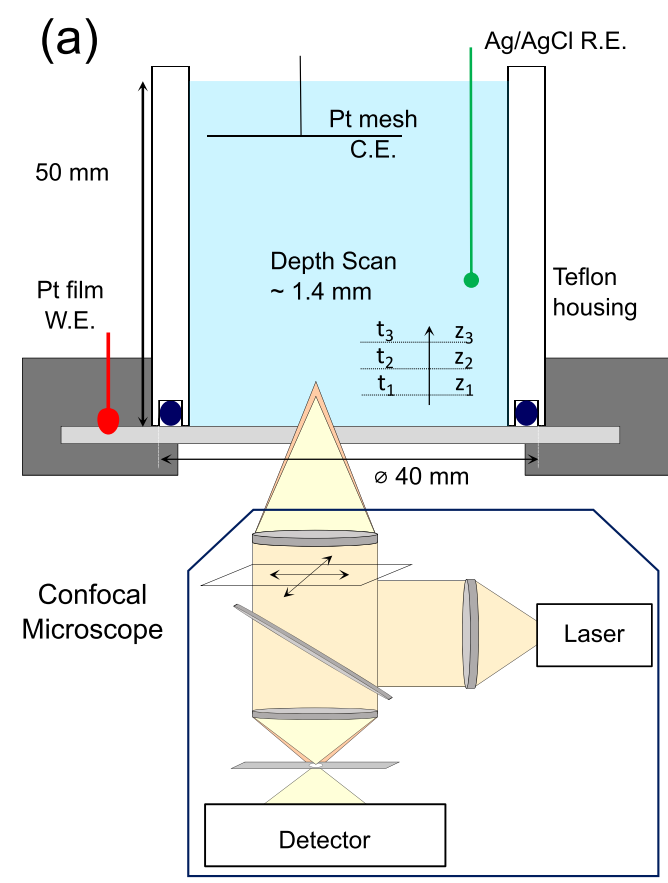

(b)

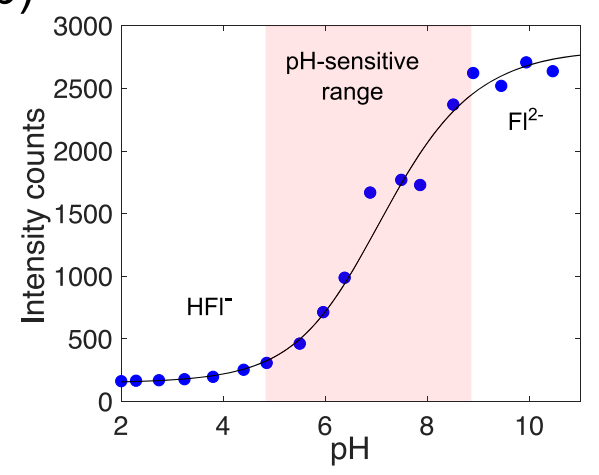

Figure 1. (a) Schematic of the experimental setup. The electrochemical cell was placed on the inverted confocal laser scanning microscope. The transparent working electrode allowed for depthwise $(z)$ measurement of fluorescent intensity. (b) Calibration results for the $\mathrm{pH}$ dependence of fluorescein. The experimental data (filled circles) shown here are the mean of three measurements of intensity measurements at each $\mathrm{pH}$ (error bars are smaller than the marker size). A sigmoidal function (black line; see Supporting Information for details of fit) is fitted to all three measurements at each $\mathrm{pH}$. The measurements were performed in $1 \mathrm{mM} \mathrm{Na}_{2} \mathrm{SO}_{4}$ containing $8 \mu \mathrm{M}$ fluorescein. $\mathrm{pH}$ was adjusted to the required value by addition of $\mathrm{H}_{2} \mathrm{SO}_{4}$.

reaction-diffusion model for the simulations of the general form

$$
\frac{\partial c_{k}}{\partial t}=D_{k} \frac{\partial^{2} c_{k}}{\partial^{2} z} \pm f(c)
$$

where $c_{k}(z, t)$ is the concentration of species $k, D_{k}$ the corresponding diffusion constant, and $f(c)$ a nonlinear function representing reaction terms. Here the chemical reactions considered are

$$
\mathrm{H}^{+}+\mathrm{OH}^{-} \underset{k_{\mathrm{b}}}{\stackrel{k_{\mathrm{f}}}{\rightleftharpoons}} \mathrm{H}_{2} \mathrm{O}
$$




$$
\mathrm{H}^{+}+\mathrm{Fl}^{2-} \underset{k_{\mathrm{b}, \mathrm{Fl}}}{\stackrel{k_{\mathrm{f}, \mathrm{Fl}}}{\rightleftharpoons}} \mathrm{HFl}^{-}
$$

where the equilibrium constants are $\frac{k_{\mathrm{b}}}{k_{\mathrm{f}}}=K_{\mathrm{W}}$ and $\frac{k_{\mathrm{b}, \mathrm{Fl}}}{k_{\mathrm{f}, \mathrm{Fl}}}=K_{\mathrm{f}, \mathrm{eq}}$. The $\mathrm{pK}_{\mathrm{a}}$ of $\mathrm{H}_{2} \mathrm{Fl}$ is lower than the $\mathrm{pH}$ considered here, and therefore, it can be ignored. Assuming that the concentration of water is large and therefore essentially constant during the experiment, the equations have been solved for the concentration of three species: $\mathrm{H}^{+}, \mathrm{OH}^{-}$, and $\mathrm{Fl}^{2-}$. Further details of the exact reaction-diffusion equations (with their boundary conditions) and the numerical technique (including validation) are presented in the Supporting Information. The contribution of capacitive current has also been taken into account. However, because the exact value of the capacitance (C) is not known in our measurements, results for $C$ values in the range $0 \leq C \leq 120 \mu \mathrm{F} / \mathrm{cm}^{2}$ are also presented in Figure 3c. Additional details can be found in the Supporting Information.

Time-Resolved $\mathrm{pH}$ Measurements. A cyclic voltammogramm (CV) of an $\mathrm{O}_{2}$ saturated solution $\left(\mathrm{pH} 5 \mathrm{HClO}_{4}+0.5 \mathrm{M}\right.$ $\mathrm{NaClO}_{4}+8 \mu \mathrm{M} \mathrm{Fl}$ ), along with the measured potential of the $\mathrm{Pt}$ working electrode for some of the constant current experiments, is shown in Figure 2. For reference, an additional
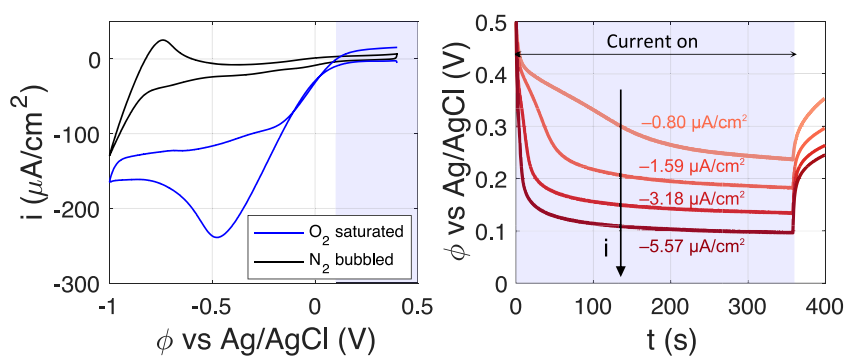

Figure 2. Left: First cycle of the cyclic voltammogram (CV) measured at $10 \mathrm{mV} / \mathrm{s}$ for the $\mathrm{Pt}$ working electrode in $\mathrm{O}_{2}$ saturated and $\mathrm{N}_{2}$ bubbled solutions ( $\left.\mathrm{pH} 5 \mathrm{HClO}_{4}+0.5 \mathrm{M} \mathrm{NaClO}_{4}+8 \mu \mathrm{M} \mathrm{Fl}\right)$ in our setup. The shaded region shows the potential range measured in chronopotentiometric (CP) experiments. Right: The CP curves obtained for the $\mathrm{O}_{2}$ saturated case. The shaded region indicates the time over which the constant current is applied. The corresponding current density for each curve is mentioned as well.

$\mathrm{CV}$ is included for the same configuration but using $\mathrm{N}_{2}$ bubbled electrolyte instead. For the current densities, $i$, and run-times considered here, it is estimated (using an initial concentration corresponding to 1 atm $\mathrm{O}_{2}$ pressure) that the oxygen at the electrode surface never gets completely depleted. This is also evident from the differences between the CV's of the $\mathrm{O}_{2}$-saturated and $\mathrm{N}_{2}$-bubbled solutions in Figure 2. Hence, it can be concluded that oxygen reduction, and not water or proton reduction, is the primary reaction occurring at the electrode. The measured potential window of operation in our constant current experiments is between 0.1 and $0.5 \mathrm{~V}$ vs $\mathrm{Ag} /$ $\mathrm{AgCl}$ (at a starting $\mathrm{pH}$ of 5), which translates to $0.6-1.1 \mathrm{~V}$ vs RHE. Given that an ovepotential of $|\Delta \phi| \gtrsim 0.3 \mathrm{~V}^{19}$ (over the thermodynamic potential of $1.23 \mathrm{~V}$ vs RHE) is required to drive the $\mathrm{O}_{2}$ reduction reaction on platinum, the potentials measured in our experiments are consistent with $\mathrm{O}_{2}$ reduction occurring at the electrode. However, because the $\mathrm{O}_{2}$ reduction reaction depends on the $\mathrm{pH}$ of the solution, ${ }^{20,21}$ the proper flux boundary conditions for $\mathrm{OH}^{-}$and $\mathrm{H}^{+}$are complicated (consumption of $\mathrm{H}^{+}$or production of $\mathrm{OH}^{-}$, depending on the reaction). Nevertheless, the calculated $\mathrm{pH}$ profiles shown here were found to be independent of this. Finally, it is important to mention that fluorescein is stable under the conditions applied. ${ }^{22-24}$

The obtained emission intensity profiles and the resulting $\mathrm{pH}$ distributions (at $0.5 \mathrm{M} \mathrm{NaClO}_{4}$ supporting electrolyte concentration) are summarized in Figure $3 \mathrm{a}$ for various (constant) applied current densities. In all cases considered here, $i$ is limited to values traditionally considered minute for electrochemistry. Despite such low current densities, the $\mathrm{pH}$ change and the corresponding thickness of the depletion layer are significant. Figure 3a shows the attenuation-corrected mean intensity of fluorescein emission as a function of distance $z$ from the electrode surface. Independent of the applied current density, a steep front is seen to propagate into the solution already at early times, $t<300 \mathrm{~s}$. This feature also translates to the corresponding $\mathrm{pH}$ profiles. It should be noted, however, that intensity levels within the resulting "shoulder" close to the electrode reach the saturation limit and because of the uncertainties described above, $\mathrm{pH}$ results are grayed out in these instances. Nevertheless, the experimental results are in good agreement with the simulated $\mathrm{pH}$ profiles shown in Figure $3 \mathrm{~b}$ (see solid lines). Interestingly, also in the simulations the $\mathrm{pH}$ is near constant close to the electrode for $|i| \geq 1.59 \mu \mathrm{A}$ / $\mathrm{cm}^{2}$, yet with $\mathrm{pH} 9-10$, the values are slightly outside the experimental sensitivity range. Even at current densities of $\sim 1$ $\mu \mathrm{A} / \mathrm{cm}^{2}$, the depletion layer or the penetration depth of the $\mathrm{pH}$ profile reaches $\sim 1 \mathrm{~mm}$ into the electrolyte. At higher current densities, this depletion length grows faster and extends further into the bulk of the solution.

It can be seen, however, that for the two highest current densities considered here, the intensity as well as the $\mathrm{pH}$ profiles recede at later times (corresponding to darker shadings of the markers), whereas the model predicts a monotonic outward propagation of the front. To enable a quantitative comparison, we track the position $z_{\mathrm{pH}} 7$ at which $\mathrm{pH} 7$ is encountered as a proxy for the front location. As Figure $3 \mathrm{c}$ shows, the $\mathrm{pH}$ front propagation in the experiments is well captured by the model for the two lower current density cases presented here. At higher current densities and at late times, though, the $\mathrm{pH}$ front in experiments either recedes or saturates. This is also true for repeat measurements made (see the Supporting Information). However, this effect appears to be an artifact of the way the mean fluorescein intensities are calculated. Consistent with the $1 \mathrm{D}$ assumption, only a measure of the mean across the entire image (i.e., a plane parallel to the electrode) is considered. For example, at $t_{1}$ (for $|i|=5.59 \mu \mathrm{A}$ / $\mathrm{cm}^{2}$, see Figure $3 \mathrm{a}$ ), this is appropriate as highlighted in Figure 4a. At $t_{2}$ though, the intensity distribution displayed in Figure $4 \mathrm{a}$ becomes distinctly inhomogeneous as seen in Figure $4 \mathrm{~b}$. This implies that $2 \mathrm{D}$ or $3 \mathrm{D}$ effects become relevant, which are not captured in the one-dimensional model.

To determine the location and time at which $3 \mathrm{D}$ effects become relevant, we consider the standard deviation $(\sigma)$ normalized with the mean intensity $(\mu)$ of the image as shown in Figure 4c. To minimize the effect of high-frequency spatial noise, the image was box-filtered with a filter size of 50 pixels before calculating $\sigma$. Figure $4 \mathrm{c}$ captures the uniform image intensity for $|i|=0.8 \mu \mathrm{A} / \mathrm{cm}^{2}$ as a near constant $\sigma / \mu$. In contrast, a visible peak in $\sigma / \mu$ at the depletion front $z=z_{\mathrm{pH} 7}$ is observed for all other cases. At the two highest current densities considered, the unsteadiness in fluorescein intensity develops over time as well. The onset time $\left(t_{\text {ons }}\right.$ defined as $\sigma / \mu$ $>0.1$ ) of this instability thus calculated is, in Figure $4 d$, found 


\section{(a) Experiments}

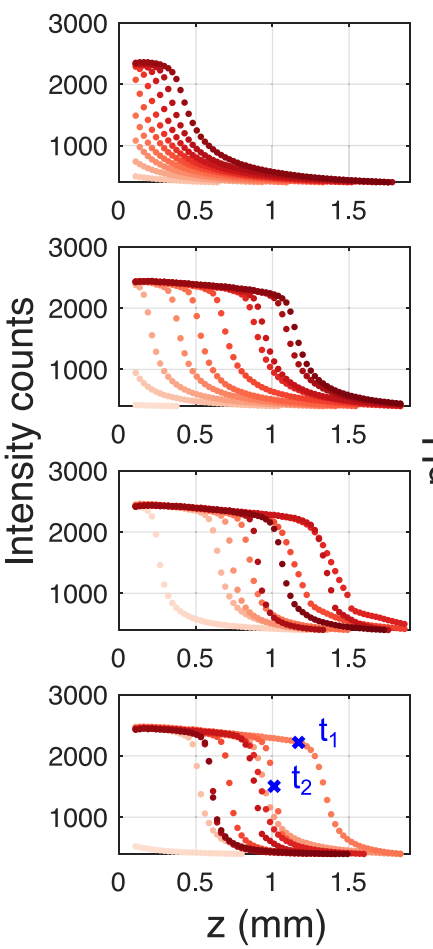

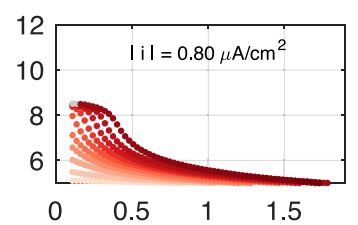
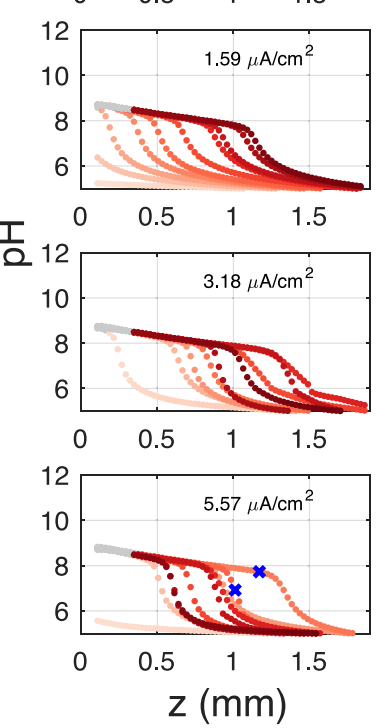

(b) Model

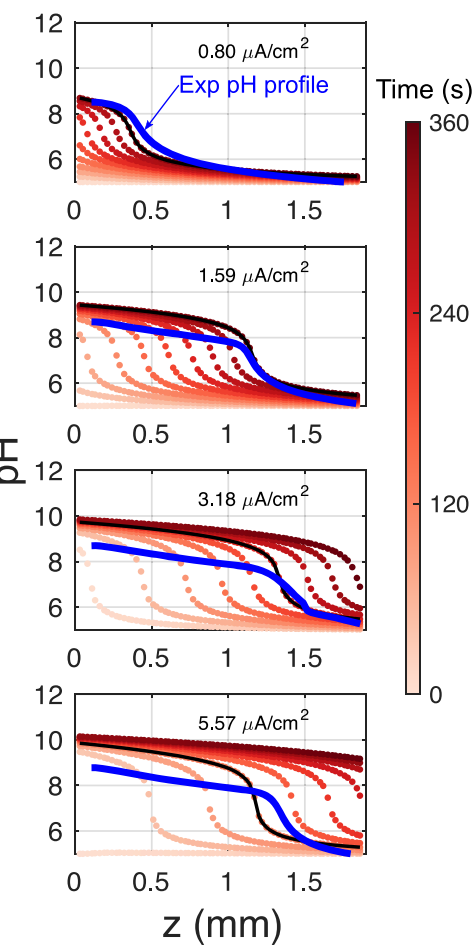

(c) Depletion length
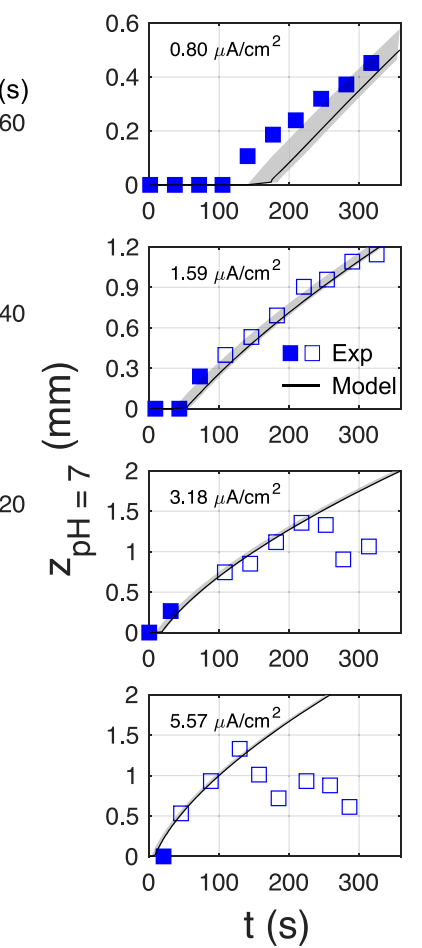

Figure 3. Experimental versus model results. The experimental measurement is restricted to $z>0.1 \mathrm{~mm}$ because of limitations of the optical setup (see Supporting Information for details). (a) Attenuation and depth-corrected fluorescein intensity profiles. Corresponding pH profiles calculated using the calibration curve. $\mathrm{pH}>8.5$ has been grayed out because of the uncertainty in the measurement at these values. The times $t_{1}$ and $t_{2}$ have been marked for use in Figure 4. (b) Model results (with $C=88 \mu \mathrm{F} / \mathrm{cm}^{2}$ ) calculated at experimental times. The solid lines are drawn to compare $\mathrm{pH}$ profiles of the experiment (blue) with the model (black). (c) Comparison of depletion length $z_{\mathrm{pH}} 7$ of experiment versus model. The shaded region indicates the model results over a range of capacitance $0 \leq C \leq 120 \mu \mathrm{F} / \mathrm{cm}^{2}$ (solid line with $C=88 \mu \mathrm{F} / \mathrm{cm}^{2}$ ). Open squares show the location of the $\mathrm{pH}$ front after the first appearance of the inhomogeneity as shown in Figure 4.

to sharply reduce with increasing current densities which is well approximated by an inverse proportionality. It is conceivable then that this instability occurs only after a certain threshold number of $\mathrm{H}^{+}$ions have been depleted from the solution. The distance $\delta$ at which this nonuniformity is first measured shows no clear trend: the nonuniformity first increases until $|i|=3.18 \mu \mathrm{A} / \mathrm{cm}^{2}$ and then decreases again slightly later. Because we look at only a small portion of the electrode though, deviations from a 1D profile can occur much earlier, at a different $\delta$. It is unlikely that the reaction at the counter electrode plays any role in the appearance of instability as it is sufficiently far compared to the measured depletion lengths of $\sim 2 \mathrm{~mm}$. Possible reasons could then be the presence of electric field effects or induced fluid flow in the system, ${ }^{25}$ which have not been modeled. However, despite the early appearance of inhomogeneity, the $\mathrm{pH}$ profiles in experiments are similar to the model results up to distances and times that are much larger (see Figure 3c, filled and open symbols, and blue/black lines in Figure $3 \mathrm{~b}$ ). It may be possible then that the departure of $\mathrm{pH}$ profiles in experiments, from a $1 \mathrm{D}$ diffusion approximation, occurs only after a certain minimum $\sigma / \mu$ (and corresponding inhomogeneity) is reached.

Sulfate Buffer Effect. In addition to the above measurements, we proceed to evaluate the developing $\mathrm{pH}$ profiles in sulfatecontaining electrolytes, e.g., in the $\mathrm{Na}_{2} \mathrm{SO}_{4} / \mathrm{H}_{2} \mathrm{SO}_{4}$ system. This system is frequently used (for example sulfuric acid is commonly used to study $\mathrm{O}_{2}$ reduction) but, in contrast to perchlorate electrolytes, may induce additional buffer capacity, thus changing the $\mathrm{pH}$ profiles. In fact, $\mathrm{H}_{2} \mathrm{SO}_{4}$ has two dissociation constants; the second corresponds to the dissociation of $\mathrm{HSO}_{4}{ }^{-}$with a $\mathrm{pK}$ a of around $2 .^{26-28}$ Figure 5 compares the $\mathrm{pH}$ profiles measured for the sulfate case to those obtained with perchlorate electrolyte, for the two lowest current densites. It is evident that the $\mathrm{pH}$ profiles develop significantly slower in sulfate-containing electrolytes. For example, for $|i|=1.59 \mu \mathrm{A} / \mathrm{cm}^{2}$, the $\mathrm{pH}$ profiles in the $\mathrm{Na}_{2} \mathrm{SO}_{4} / \mathrm{H}_{2} \mathrm{SO}_{4}$ system have no clear front propagating in the solution; the profiles rather become increasingly steep close to the electrode surface with time, while for the perchlorate solution depletion lengths of $z_{\mathrm{pH}} \approx 1.5 \mathrm{~mm}$ are achieved.

To try to further explain the experimental results, we consider the $\mathrm{pK}_{\mathrm{a}}$ of $\mathrm{HSO}_{4}{ }^{-}$, which, although it is well below our starting $\mathrm{pH}(\mathrm{pH} \mathrm{5})$, because of the presence of the large concentration of $\mathrm{SO}_{4}^{2-}$ in solution, creates a reservoir of $\mathrm{HSO}_{4}{ }^{-}$ions which acts as a source of protons in solution and stabilizes the solution against $\mathrm{pH}$ changes. We attempt to capture this effect in the $1 \mathrm{D}$ model as our results in the Supporting Information show. This buffer effect is most likely present in experimental measurements in the literature with sulfate electrolytes. ${ }^{5,9}$ For example, Leenheer and Atwater ${ }^{9}$ measured the $\mathrm{pH}$ on patterned gold electrodes in $\mathrm{Na}_{2} \mathrm{SO}_{4}$ solutions, with different pattern shapes and area. However, their steady-state simulations predicted a depletion zone much larger than experiments. Similarly, the buffering effect of $\mathrm{Li}_{2} \mathrm{SO}_{4}$ solutions may also be present in the recent measurements by Monteiro et al. ${ }^{5}$ A comparison such as ours, between 


\section{(a) Homogenous}

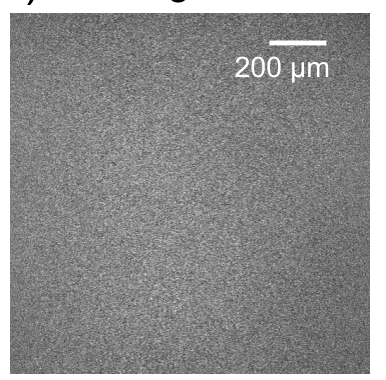

(b) Inhomogenous

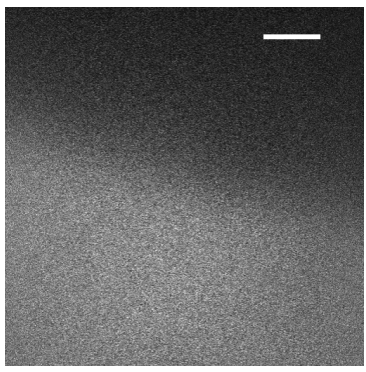

(c) Identifiying Inhomogenity

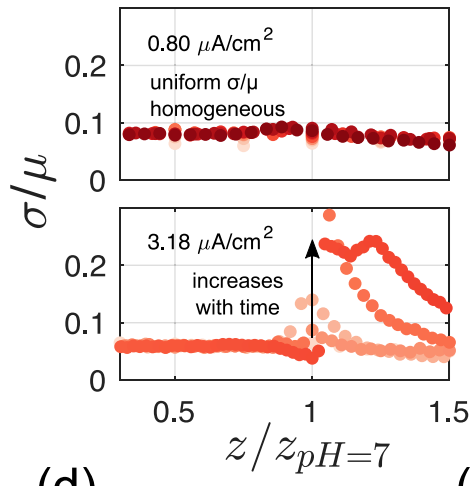

(d)

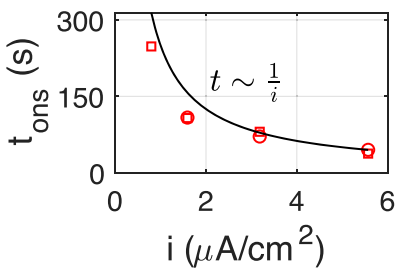

(e)

(e)
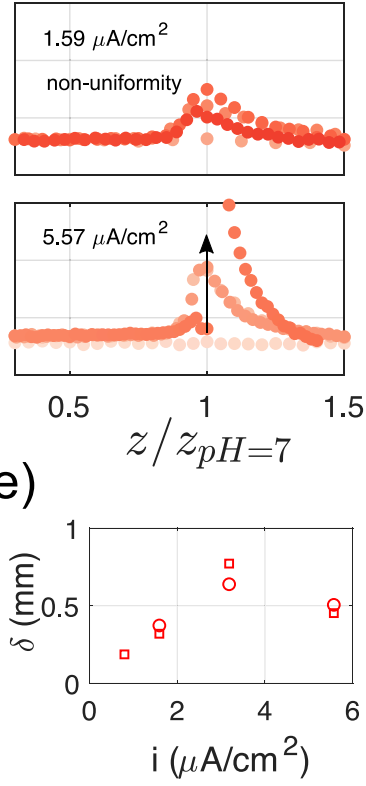

Figure 4. Inhomogeneous fluorescein intensity in a plane. Example of fluorescein intensity images $\left(|i|=5.59 \mu \mathrm{A} / \mathrm{cm}^{2}\right)$ at times marked in Figure 3a: (a) homogeneous image at time $t_{1}$ and (b) inhomogeneous at time $t_{2}$. (c) $\sigma / \mu$ versus depth for all current densities. The same color bar as in Figure 3 applies. Sharp changes are used to pick out times and positions where this nonuniformity is observed. (d) The onset time $t_{\text {ons }}$ and (e) the location $\delta$ at which the inhomogeneity is first observed vs current density. Different symbols are repeat measurements. The solid line in panel $\mathrm{d}$ corresponds to $t_{\mathrm{ons}}=\frac{250}{i} \mathrm{~s}$ and is arbitrarily chosen to highlight the inverse relationship between $t_{\text {ons }}$ and $i$.

perchlorate and sulfate electrolytes, should help to quantify the magnitude of this effect and help better interpret results.

To summarize, we have successfully demonstrated the use of fluorescein to measure time-resolved $\mathrm{pH}$ profiles in solution. The results of a time-dependent reaction-diffusion model compare reasonably well with the experimental data. However, the inhomogeneity of $\mathrm{pH}$ in a plane that develops at "high currents" clearly shows the need for time-varying local $\mathrm{pH}$ measurements. The crucial aspects to consider when using fluorescence microscopy for $\mathrm{pH}$ measurement, like optical distortions and signal attenuation, have been carefully examined. Furthermore, the concentration of the supporting electrolyte is shown to influence migration of fluorescent dyes and should be considered to avoid pitfalls in $\mathrm{pH}$ measurement in electrochemical systems. For sulfate-containing electrolytes, our analysis reveals buffering effects, which likely explain the (a) $\mathrm{HClO}_{4} / \mathrm{NaClO}_{4}$
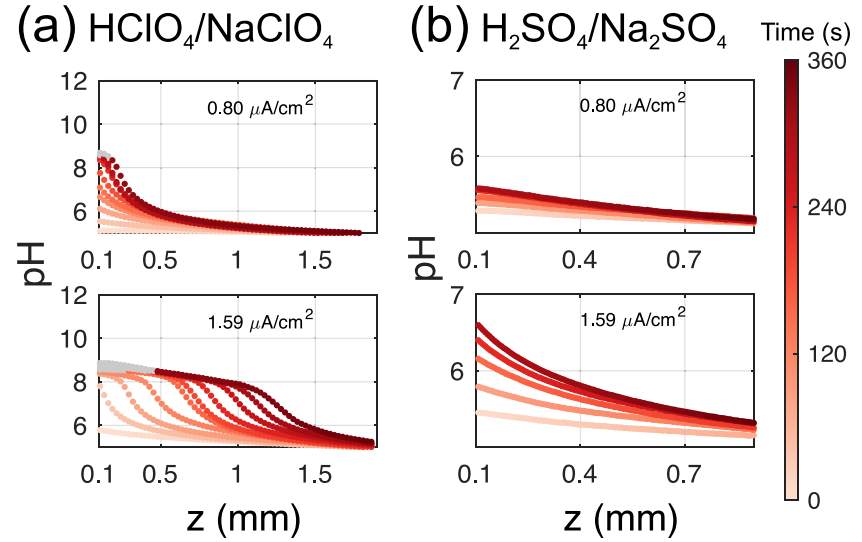

Figure 5. Comparison of $\mathrm{pH}$ profiles for (a) perchlorate $\left(\mathrm{NaClO}_{4} /\right.$ $\left.\mathrm{HClO}_{4}\right)$ and (b) sulfate $\left(\mathrm{Na}_{2} \mathrm{SO}_{4} / \mathrm{H}_{2} \mathrm{SO}_{4}\right)$ electrolytes. All measurements were performed in $0.5 \mathrm{M}$ supporting salt concentration containing $8 \mu \mathrm{M} \mathrm{Fl}$. $\mathrm{pH}$ was adjusted to $\mathrm{pH} 5$ by addition of respective acid. All solutions were bubbled with $\mathrm{N}_{2}$ before starting the experiment. $\mathrm{pH}>8.5$ has been grayed out because of the uncertainty in the measurements at these values.

difference between the measured diffusion profiles and those observed in experiments in the past. ${ }^{9}$

Fluorescence microscopy offers time-resolved and relatively nonintrusive measurement of $\mathrm{pH}$ instantly over a large area. Because the principle of measurement presented here is applicable to other fluorescent dyes with a different $\mathrm{pH}$ detection range, this technique can be used for a wide range of electrochemical systems to elucidate electrode dynamics. This holds in particular for $\mathrm{CO}_{2}$ reduction on gas diffusion electrodes, because the second $\mathrm{p} K_{\mathrm{a}}$ of carbonic acid lies in the fluorescein detection region. Our developed method can be directly implemented to quantify mass transport, the role of bicarbonate concentrations, etc. in the electrolyte. More generally, the measurement technique presented here offers insight into the dynamics of ions in solution, important to many electrochemical systems, none more so than in electrochemical cells to unravel the role of start-stop transients. Detailed information on the $\mathrm{pH}$ distribution will provide a better understanding of electrode processes and aid in the overall design of electrochemical systems for eventual use in large-scale electrolysis.

\section{EXPERIMENTAL METHODS}

For the working electrode, a $3 \mathrm{~nm}$ thick chromium (under) layer was used for better adhesion between the platinum film and the glass slide. The sheet resistance of the resulting thinfilm electrode was $69 \Omega$. The electrical connection to the working electrode was made with a platinized titanium point contact. Prior to the measurement, the $\mathrm{pH}$ of the solution was adjusted to a $\mathrm{pH}$ value of 5 by addition of appropriate amounts of $0.1 \mathrm{M} \mathrm{HClO}_{4}$ (or $0.5 \mathrm{M} \mathrm{H}_{2} \mathrm{SO}_{4}$ for sulfate electrolytes). The $\mathrm{pH}$ of the solution before the start of each experiment (as well as the calibration solutions shown in Figure 1b) was measured using the Hannah Instruments Edge-pH meter that has an accuracy of $\pm 0.02 \mathrm{pH}$ units. All chemicals were purchased from Sigma-Aldrich. Further experimental and numerical details can be found in the Supporting Information. 


\section{ASSOCIATED CONTENT}

\section{(5) Supporting Information}

The Supporting Information is available free of charge at https://pubs.acs.org/doi/10.1021/acs.jpclett.0c01575.

Details of experimental method, numerical method, repeat measurements and comparison of experiments, and model for sulfates (PDF)

\section{AUTHOR INFORMATION}

\section{Corresponding Authors}

Nakul Pande - Physics of Fluids and Photo Catalytic Synthesis, University of Twente, Enschede, The Netherlands;

Email: n.pande@utwente.nl

Bastian T. Mei - Photo Catalytic Synthesis, University of Twente, Enschede, The Netherlands; (1) orcid.org/0000-00023973-9254; Email: b.t.mei@utwente.nl

Dominik Krug - Physics of Fluids, University of Twente, Enschede, The Netherlands; (1) orcid.org/0000-0002-06275676; Email: d.j.krug@utwente.nl

\section{Authors}

Shri K. Chandrasekar - Photo Catalytic Synthesis, University of Twente, Enschede, The Netherlands

Detlef Lohse - Physics of Fluids, University of Twente, Enschede,

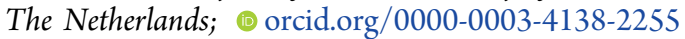

Guido Mul - Photo Catalytic Synthesis, University of Twente, Enschede, The Netherlands; 10 orcid.org/0000-0001-58986384

Jeffery A. Wood - Soft Matter, Fluidics and Interfaces, University of Twente, Enschede, The Netherlands; 이이. ord. 0000-0002-9438-1048

Complete contact information is available at:

https://pubs.acs.org/10.1021/acs.jpclett.0c01575

\section{Notes}

The authors declare no competing financial interest.

\section{ACKNOWLEDGMENTS}

This research received funding from The Netherlands Organization for Scientific Research (NWO) in the framework of the fund New Chemical Innovations, project ELECTROGAS (731.015.204), with financial support of Akzo Nobel Chemicals, Shell Global Solutions, Magneto Special Anodes (an Evoqua Brand), and Elson Technologies. We acknowledge The Netherlands Center for Multiscale Catalytic Energy Conversion (MCEC) and the Max Planck Center Twente for Complex Fluid Dynamics for financial support. D.L. also acknowledges financial support by an ERC-Advanced Grant.

\section{REFERENCES}

(1) Pourbaix, M.; Zhang, H.; Pourbaix, A. Presentation of an Atlas of Chemical and Electrochemical Equilibria in the Precence of a Gaseous Phase. Mater. Sci. Forum 1997, 251-254, 143-148.

(2) Kas, R.; Kortlever, R.; Y1lmaz, H.; Koper, M. T. M.; Mul, G. Manipulating the Hydrocarbon Selectivity of Copper Nanoparticles in $\mathrm{CO}_{2}$ Electroreduction by Process Conditions. ChemElectroChem 2015, 2, 354-358.

(3) Yang, K.; Kas, R.; Smith, W. A. Tenacious Mass Transfer Limitations Drive Catalytic Selectivity during Electrochemical Carbon Dioxide Reduction. 2019, ChemRxiv

(4) Burdyny, T.; Smith, W. A. $\mathrm{CO}_{2}$ reduction on gas-diffusion electrodes and why catalytic performance must be assessed at commercially-relevant conditions. Energy Environ. Sci. 2019, 12, $1442-1453$

(5) Monteiro, M. C. O.; Jacobse, L.; Touzalin, T.; Koper, M. T. M. Mediator-Free SECM for Probing the Diffusion Layer $\mathrm{pH}$ with Functionalized Gold Ultramicroelectrodes. Anal. Chem. 2020, 92, $2237-2243$.

(6) Bouffier, L.; Doneux, T. Coupling electrochemistry with in situ fluorescence (confocal) microscopy. Curr. Opin. Electrochem. 2017, 6, $31-37$.

(7) Cannan, S.; Douglas Macklam, I.; Unwin, P. R. Threedimensional imaging of proton gradients at microelectrode surfaces using confocal laser scanning microscopy. Electrochem. Commun. 2002, 4, 886-892.

(8) Rudd, N. C.; Cannan, S.; Bitziou, E.; Ciani, I.; Whitworth, A. L.; Unwin, P. R. Fluorescence Confocal Laser Scanning Microscopy as a Probe of $\mathrm{pH}$ Gradients in Electrode Reactions and Surface Activity. Anal. Chem. 2005, 77, 6205-6217.

(9) Leenheer, A. J.; Atwater, H. A. Imaging Water-Splitting Electrocatalysts with $\mathrm{pH}$-Sensing Confocal Fluorescence Microscopy. J. Electrochem. Soc. 2012, 159, H752-H757.

(10) Mai, J.; Miller, H.; Hatch, A. V. Spatiotemporal Mapping of Concentration Polarization Induced $\mathrm{pH}$ Changes at Nanoconstrictions. ACS Nano 2012, 6, 10206-10215.

(11) Andersen, M. B.; Rogers, D. M.; Mai, J.; Schudel, B.; Hatch, A. V.; Rempe, S. B.; Mani, A. Spatiotemporal pH Dynamics in Concentration Polarization near Ion-Selective Membranes. Langmuir 2014, 30, 7902-7912.

(12) Pande, N.; Mul, G.; Lohse, D.; Mei, B. Correlating the ShortTime Current Response of a Hydrogen Evolving Nickel Electrode to Bubble Growth. J. Electrochem. Soc. 2019, 166, E280-E285.

(13) Angulo, A.; van der Linde, P.; Gardeniers, H.; Modestino, M.; Fernández Rivas, D. Influence of Bubbles on the Energy Conversion Efficiency of Electrochemical Reactors. Joule 2020, 4, 555-579.

(14) Bowyer, W. J.; Xie, J.; Engstrom, R. C. Fluorescence Imaging of the Heterogeneous Reduction of Oxygen. Anal. Chem. 1996, 68, 2005-2009.

(15) Tassy, B.; Dauphin, A. L.; Man, H. M.; Le Guenno, H.; Lojou, E.; Bouffier, L.; de Poulpiquet, A. In Situ Fluorescence Tomography Enables a 3D Mapping of Enzymatic $\mathrm{O}_{2}$ Reduction at the Electrochemical Interface. Anal. Chem. 2020, 92, 7249-7256.

(16) Diehl, H.; Markuszewski, R. Studies on fluorescein-II: The solubility and acid dissociation constants of fluorescein in water solution. Talanta 1985, 32, 159-165.

(17) Diehl, H.; Markuszewski, R. Studies on fluorescein-VII: The fluorescence of fluorescein as a function of $\mathrm{pH}$. Talanta 1989, 36, $416-418$.

(18) Sjöback, R.; Nygren, J.; Kubista, M. Absorption and fluorescence properties of fluorescein. Spectrochim. Acta, Part A 1995, 51, L7-L21.

(19) Li, M. F.; Liao, L. W.; Yuan, D. F.; Mei, D.; Chen, Y.-X. pH effect on oxygen reduction reaction at $\mathrm{Pt}(111)$ electrode. Electrochim. Acta 2013, 110, 780-789.

(20) Si, F.; Zhang, Y.; Yan, L.; Zhu, J.; Xiao, M.; Liu, C.; Xing, W.; Zhang, J. Rotating Electrode Methods and Oxygen Reduction Electrocatalysts; Elsevier, 2014; pp 133-170.

(21) Pletcher, D.; Sotiropoulos, S. A study of cathodic oxygen reduction at platinum using microelectrodes. J. Electroanal. Chem. 1993, 356, 109-119.

(22) Compton, R. G.; Daly, P. J.; Unwin, P. R.; Waller, A. M. In-situ electrochemical ESR: ECE versus dispi. J. Electroanal. Chem. Interfacial Electrochem. 1985, 191, 15-29.

(23) Compton, R. G.; Harland, R. G.; Unwin, P. R.; Waller, A. M. Rotating-disc electrodes. ECE and DISP1 processes. J. Chem. Soc., Faraday Trans. 1 1987, 83, 1261.

(24) Compton, R. G.; Mason, D.; Unwin, P. R. The reduction of fluorescein in aqueous solution (at $\mathrm{pH} 6$ ). A new DISP2 reaction. J. Chem. Soc., Faraday Trans. 1 1988, 84, 483. 
(25) Mani, A.; Wang, K. M. Electroconvection Near Electrochemical Interfaces: Experiments, Modeling, and Computation. Annu. Rev. Fluid Mech. 2020, 52, 509-529.

(26) Hamer, W. J. The Ionization Constant and Heat of Ionization of the Bisulfate Ion from Electromotive Force Measurements 1. J. Am. Chem. Soc. 1934, 56, 860-864.

(27) Covington, A. K.; Dobson, J. V.; Wynne-Jones, L. Dissociation constant of the bisulphate ion at $25^{\circ} \mathrm{C}$. Trans. Faraday Soc. 1965, 61, 2057-2062.

(28) Wu, Y. C.; Feng, D. The second dissociation constant of sulfuric acid at various temperatures by the conductometric method. $J$. Solution Chem. 1995, 24, 133-144. 